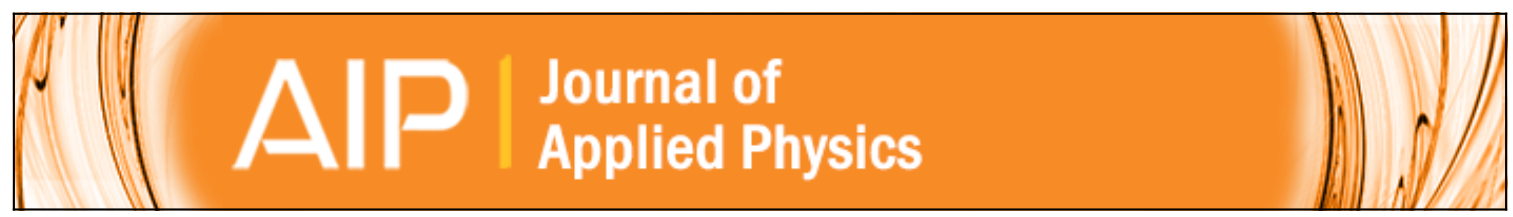

Calculation of the temperature dependence of the giant MR and application to Co/Cu multilayers

J. L. Duvail, A. Fert, L. G. Pereira, and D. K. Lottis

Citation: Journal of Applied Physics 75, 7070 (1994); doi: 10.1063/1.356729

View online: http://dx.doi.org/10.1063/1.356729

View Table of Contents: http://scitation.aip.org/content/aip/journal/jap/75/10?ver=pdfcov

Published by the AIP Publishing

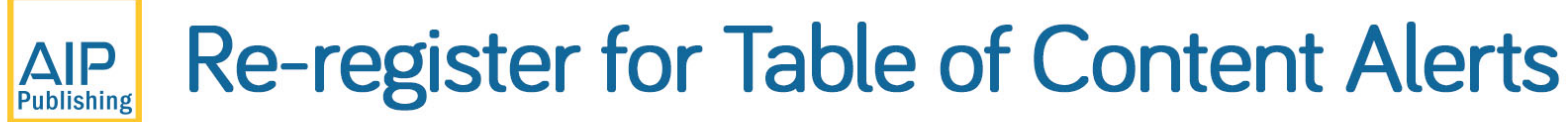

Create a profile.

Sign up today! 


\title{
Calculation of the temperature dependence of the giant MR and application to $\mathrm{Co} / \mathrm{Cu}$ multilayers
}

\author{
J. L. Duvail, A. Fert, and L. G. Pereira \\ Laboratoire de Physique des Solides, Bât 510, Université Paris-Sud, 91405 Orsay, France \\ D. K. Lottis \\ School of Physics and Astronomy, University of Minnesota, Minneapolis, Minnesota 55455
}

\begin{abstract}
Most theoretical models of the giant magnetoresistance (GMR) in metallic magnetic multilayers developed up to now are for the zero-temperature limit, thus neglecting the spin-flip scattering arising from spin fluctuations (magnons), as well as other scatterings from thermal excitations. To account for the temperature dependence of the GMR, we have introduced electron-magnon and electron-phonon scattering terms in a Camley-Barnas-like semi-classical model. We apply our calculation to the interpretation of the temperature dependence of the resistivity and GMR in $\mathrm{Co} / \mathrm{Cu}$.
\end{abstract}

\section{INTRODUCTION}

The giant magnetoresistance (GMR) of the magnetic multilayers, first discovered in $\mathrm{Fe} / \mathrm{Cr}$ structures, ${ }^{1}$ has now been observed in many systems. Its interpretation ${ }^{2-4}$ is generally based on the simplest version of the two current model for the conduction in ferromagnetic metals. ${ }^{5}$ One assumes that the electrical current is carried in independent channels by the spin $\uparrow$ (majority spin direction) and spin $\downarrow$ (minority) electrons, which is the low temperature limit of the two current model. However, at finite temperature, the spin-mixing by electron-magnon scattering, which is the transfer of momentum between the two channels, becomes an essential ingredient of the two current conduction in ferromagnets. ${ }^{5}$ In multilayers, the spin-mixing effect is expected to play an important role for the temperature dependence of the GMR and must be taken into account in the interpretation at finite temperature. In the paper, we present a model of the temperature dependence of the GMR, taking into account not only the contribution from thermally excited scatterings within each channel (that is the electron-phonon scattering and momentum nonconserving part of the electron-magnon scattering called "incoherent electron-magnon scattering" in the notation of Ref. 5), but also the (interchannel) spinmixing contribution from momentum conserving (or coherent in the notation of Ref. 5) electron-magnon scattering. To our knowledge, the spin-mixing contribution (spin-flip scattering with momentum transfer) has never been taken into account in theoretical models, and only the intrachannel magnon scattering has been introduced in calculations. ${ }^{6,7}$

\section{MODEL}

We consider an infinite multilayer composed of ferromagnetic metallic layers ( $\mathrm{Co}$, for example), separated by nonmagnetic metallic layers ( $\mathrm{Cu}$, for example). The current direction is in the plane of the layers.

Our starting point for the low temperature limit is the semi-classical model of Johnson and Camley, ${ }^{8}$ who take into account interfaces scattering by introducing interfacial layers in which the mean-free path (MFP) is shorter than inside the layers. The spin asymmetry of the MFP in the interfacial layer can also be different from that within the layers. The parameters of the model of Johnson and Camley include the resistivity of the nonmagnetic metal or what is equivalent, the MFP in the nonmagnetic layers that we will call $\lambda_{\mathrm{Cu}}(4.2$ $\mathrm{K})$, in the same way the two MFP in the magnetic layers that we call $\lambda_{\mathrm{C}_{0}}^{\dagger}(4.2 \mathrm{~K})$ and $\lambda_{\mathrm{CO}_{0}}^{\downarrow}(4.2 \mathrm{~K})$ for the spin $\uparrow$ and spin $\downarrow$ electrons respectively, the MFP $\lambda \mid(4.2 \mathrm{~K})$ and $\lambda\}(4.2 \mathrm{~K})$ in the interfacial layers, the thickness of the interfacial layer $t_{\mathrm{l}}$, and the thicknesses of the effective magnetic and nonmagnetic layers $\left(t_{\mathrm{Co}^{-}} t_{\mathrm{I}}\right)$ and $\left(t_{\mathrm{Cu}^{-}} t_{\mathrm{I}}\right)$, respectively. In Table I, we have listed the parameters of our fit expressed in terms of resistivity. We also introduce the cut-off in the angular integration suggested by Vedyayev et al. ${ }^{9}$

At finite temperature, we introduce the following temperature-dependent resistivity terms.

\section{A. Within the layers}

(i) A temperature-dependent contribution is added to the resistivity of each channel, i.e., we write

$$
\rho_{\sigma}(T)=\rho_{\sigma}(4.2 \mathrm{~K})+\delta \rho_{\sigma}(T) .
$$

TABLE I. Resistivity terms used in our calculation $\rho_{C u}^{\dagger(1)}, \rho_{C o}^{\dagger(1)}$, and $\rho_{1}^{f(1)}$ are the intrachannel resistivities for the $\mathrm{Cu}, \mathrm{Co}$, and interfacial layers, respectively. Their temperature-dependent part $\delta \rho^{f(t)}(T)$ are derived from experimental data on bulk materials for $\mathrm{Cu}$ and $\mathrm{Co}$ in Refs. 10 and 11 and is a free parameter for the interfacial layer. The spin-mixing resistivity $\rho_{C o}^{t d}$ is derived from Ref. 11 , while $\rho \downarrow \downarrow$ is a free parameter. (c) and (d) refer to two types of calculation, as explained in the text. The contribution of the resistivity term to the mean-free path inverse is $\rho_{0} \lambda_{0}=\left(h k_{F} / n e^{2}\right)=1940$ (Ref. 23). All the resistivities are given in $\mu \Omega \mathrm{cm}$.

\begin{tabular}{|c|c|c|c|c|c|}
\hline$T$ & & $4.2 \mathrm{~K}$ & $100 \mathrm{~K}$ & $200 \mathrm{~K}$ & $300 \mathrm{~K}$ \\
\hline 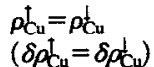 & & $\begin{array}{l}8.1 \\
(0)\end{array}$ & $\begin{array}{c}8.8 \\
(0.70)\end{array}$ & $\begin{array}{c}10.2 \\
(2.12)\end{array}$ & $\begin{array}{c}11.5 \\
(3.40)\end{array}$ \\
\hline$\rho_{C_{0}}$ & & 24.9 & 25.6 & 27.3 & 29.3 \\
\hline$\left(\delta \rho_{c_{0}}\right)$ & & $(0)$ & $(0.73)$ & $(2.47)$ & (4.42) \\
\hline$\rho_{\mathrm{C}_{0}}^{1}$ & & 176.4 & 183.0 & 198.0 & 210.9 \\
\hline$\left(\delta \rho t_{0}\right)$ & & $(0)$ & (7.3) & (22.2) & (35.3) \\
\hline$\rho_{C}^{+1}$ & & 0 & 0.55 & 2.2 & 5.0 \\
\hline$\rho \mid$ & (c) & 24.9 & 24.9 & 24.9 & 24.9 \\
\hline$\left(\delta \rho_{\mathrm{h}}^{\dagger}\right)$ & & $(0)$ & $(0)$ & (0) & $(0)$ \\
\hline$\rho \mathrm{f}$ & (d) & 24.9 & 36.2 & 63.5 & 97.9 \\
\hline$\left(\delta p_{1}^{\dagger}\right)$ & & $(0)$ & (11.3) & (38.6) & $(73.0)$ \\
\hline$\rho t$ & (c) & 485 & 485 & 485 & 485 \\
\hline$\left(\delta \rho_{1}\right)$ & & $(0)$ & $(0)$ & $(0)$ & $(0)$ \\
\hline pt & (d) & 485 & 571 & 882 & 1212 \\
\hline$\left(\delta p_{1}{ }^{\downarrow}\right)$ & & $(0)$ & (86) & (397) & (727) \\
\hline$\left.\rho\right|^{+}$ & (c) & 0 & 4.73 & 12.5 & 25.9 \\
\hline$\rho \mathrm{I}^{1}$ & (d) & 0 & 0 & 0 & 0 \\
\hline
\end{tabular}




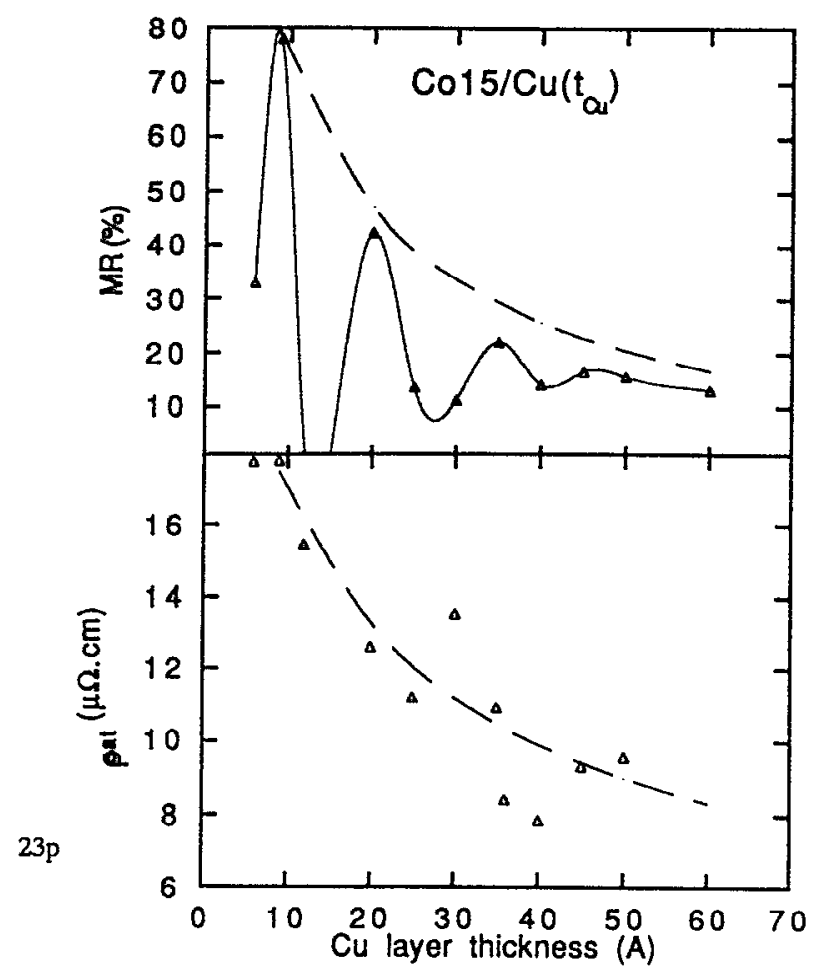

FIG. 1. Variation of the resistivity in the saturated state (bottom) and MR (top) as a function of the thickness of $\mathrm{Cu}$ for $\mathrm{Co} / \mathrm{Cu}$ multilayers. Triangles: experimental data at $4.2 \mathrm{~K}$ for (Co $15 \AA / C u) \times 30$ from Mosca et al. (Ref. 13). Dashed lines: calculation with the values of $\rho_{\mathrm{C}_{\mu}}, \rho_{\mathrm{C}_{0}}^{\uparrow}, \rho_{\mathrm{C}_{0}}^{\downarrow}, \rho_{\mathrm{A}}^{\uparrow}$, and $\rho_{\mathrm{I}}$ indicated in the text and Table I.

The intrachannel term $\delta \rho_{\sigma}(T)$ includes contributions from electron-phonon scattering and also the incoherent part of the electron-magnon $(\mathrm{e}-\mathrm{m})$ scattering transferred to the lattice. ${ }^{5}$ For $\mathrm{Cu}$, we will use the values of $\delta \rho_{\uparrow}(\mathrm{I})=\delta \rho_{\downarrow}(T)$ derived from the resistivity data of Ref. 10 for bulk $\mathrm{Cu}$. For Co, we will use values of $\delta \rho_{C_{0}}^{\dagger}$ and $\delta \rho_{C_{C}}^{\downarrow}$ derived from Ref. 10 and from resistivity measurements on Co-based dilute alloys. ${ }^{11}$ All these resistivities are listed in Table I for several temperatures between 4.2 and $300 \mathrm{~K}$.

(ii) A temperature-dependent spin-mixing term $\rho \uparrow \downarrow(T)$ is introduced to couple the Boltzmann equations of the two channels [see Eq. (3) below]. It expresses the fact that the spin-flip scattering by magnons, a part of the electron momentum is coherently transferred to the channel with opposite spin and contributes to the interchannel term $\rho \uparrow \downarrow .^{5}$ Data for $\rho_{\uparrow \downarrow}^{C_{1}}(T)$ are taken from Ref. 11 and extrapolated up to 300 $\mathrm{K}$ by assuming a variation as $T^{2}$.

\section{B. Inside the interfacial layers}

We also introduce temperature-dependent resistivity terms, which, essentially, should express the scattering by enhanced spin fluctuations at the interfaces. We take into account either intrachannel resistivity terms $\delta \rho_{\uparrow}^{\mathrm{I}}(T)$ and $\delta \rho_{\perp}^{I}(T)$, or, alternatively, an interchannel spin-mixing term $\rho_{T \downarrow}^{I}(T)$. The incoherent scattering should be predominant for a disordered interface. When the interchannel spin-mixing term is taken into account, the Boltzmann equation of the channel $\sigma$ in a given layer is written as

$$
\begin{aligned}
\frac{m}{\hbar k_{F}} & \frac{d g^{\sigma}(z, \mathbf{v})}{d z}+\frac{g^{\sigma}(z, \mathbf{v})}{\lambda^{\sigma} v_{z}}+\frac{g^{\sigma}(z, \mathbf{v})-g^{-\sigma}(z, \mathbf{v})}{\lambda !^{\top !} v_{z}} \\
& =e E \partial f_{0}(v) \\
\hbar k_{F} v_{z} \delta v_{x} &
\end{aligned}
$$

where $g^{\sigma}$ is the deviation of the electron distribution function from the Fermi-Dirac distribution $f_{0}(v)$ for the spin direction $\sigma, z$ and $x$ are the directions, respectively, perpendicular to the layers and parallel to the current, $\mathbf{v}$ is the electron velocity, $E$ is the electric field. The MFP inverses, $\left(\lambda^{\sigma}\right)^{-1}$ and $\left(\lambda^{\dagger}\right)^{-1}$, include the contributions from all the scattering mechanisms.

\section{RESULT FOR THE Co/Cu SYSTEM}

We have applied our calculation to the fit of experimental results by Mosca et al. ${ }^{13} \mathrm{NMR}^{14}$ experiments on the same samples have established that the roughness of the interfaces can be modelized by a very dense distribution of monoatomic steps, so that we fix the thickness of the interfacial layer at $2 \AA$.

At low temperature, we begin by adjusting the MFP $\lambda_{\mathrm{Cu}}^{\sigma}(4.2 \mathrm{~K}), \lambda_{\mathrm{Co}_{\mathrm{o}}}(4.2 \mathrm{~K}), \lambda_{\mathrm{Co}_{\mathrm{o}}}^{\dagger}(4.2 \mathrm{~K}), \lambda_{\mathrm{f}}^{\uparrow}(4.2 \mathrm{~K}), \lambda_{\ddagger}(4.2 \mathrm{~K})$ to fit the absolute values of the resistivity and the MR ratio for several thicknesses. In Fig. 1 we show the fit of the variation of the resistivity and MR ratio with the thickness of $\mathrm{Cu}$ obtained for the values of $\rho_{\mathrm{Cu}}^{\uparrow(\downarrow)}(4.2 \mathrm{~K}), \rho_{\mathrm{Co}}^{\uparrow(\downarrow)}(4.2 \mathrm{~K})$, and $\rho_{\mathrm{I}}^{\uparrow(\downarrow)}(4.2$ $\mathrm{K})$ listed in Table I. We estimate the error bar on the MFP less than $15 \%$.

To interpret the temperature dependence, we have focused on two samples with respectively thin $(15 \AA)$ and thick $(60 \AA)$ Co layers in order to determine the relative importance of the bulk and interface temperature-dependent scatterings. We have introduced the different temperaturedependent contributions successively.

The intra-channel terms $\delta \rho_{\mathrm{Cu}}(T)$ (derived from Ref. 10), $\delta \rho_{\mathrm{C}_{0}}^{\dagger}(T)$ and $\delta \rho_{\mathrm{Co}}^{\downarrow}(T)$ (derived from Refs. 10 and 11) are introduced first. They are not free parameters. As shown by curves (a) in Fig. 2, the contribution from these terms accounts only for a small part of the variation of the resistivity and MR ratio with $T$.

Then, in addition, we introduce the spin-mixing term $\rho_{\uparrow l}^{\mathrm{CO}}(T)$ derived from Ref. 11. As shown by the curves (b) in Fig. 2, this is still not sufficient to account for the variation with temperature, especially for the resistivity [we have tried fits with enhanced values of $\rho_{\uparrow \uparrow}^{\mathrm{Co}}(T)$, without succeeding in accounting for the $T$ dependence of the resistivity and MR ratio at the same time].

We are thus led to include $T$-dependent scattering by the interfaces, and we have begin by adding up a spin-mixed term $\rho_{\uparrow \downarrow}^{\mathrm{I}}(T)$ (the values are listed in Table I). We obtain the curves (c) in Fig. 2, which mean that we can obtain a good fit of the variation with for the resistivity, but not for the MR ratio (or vice versa).

We have then left out the term $\rho_{\uparrow(}^{\mathrm{I}}(T)$ and introduced $T$-dependent intra-channel terms $\delta \rho_{\sigma}^{\mathrm{I}}(T)$ in the interfacial layer (with the same values for the Co $15 \AA / \mathrm{Cu} 9 \AA$ and Co $60 \AA / \mathrm{Cu} 9 \AA$ samples). We could obtain a good agreement for the MR ratio of both samples and a good agreement for 

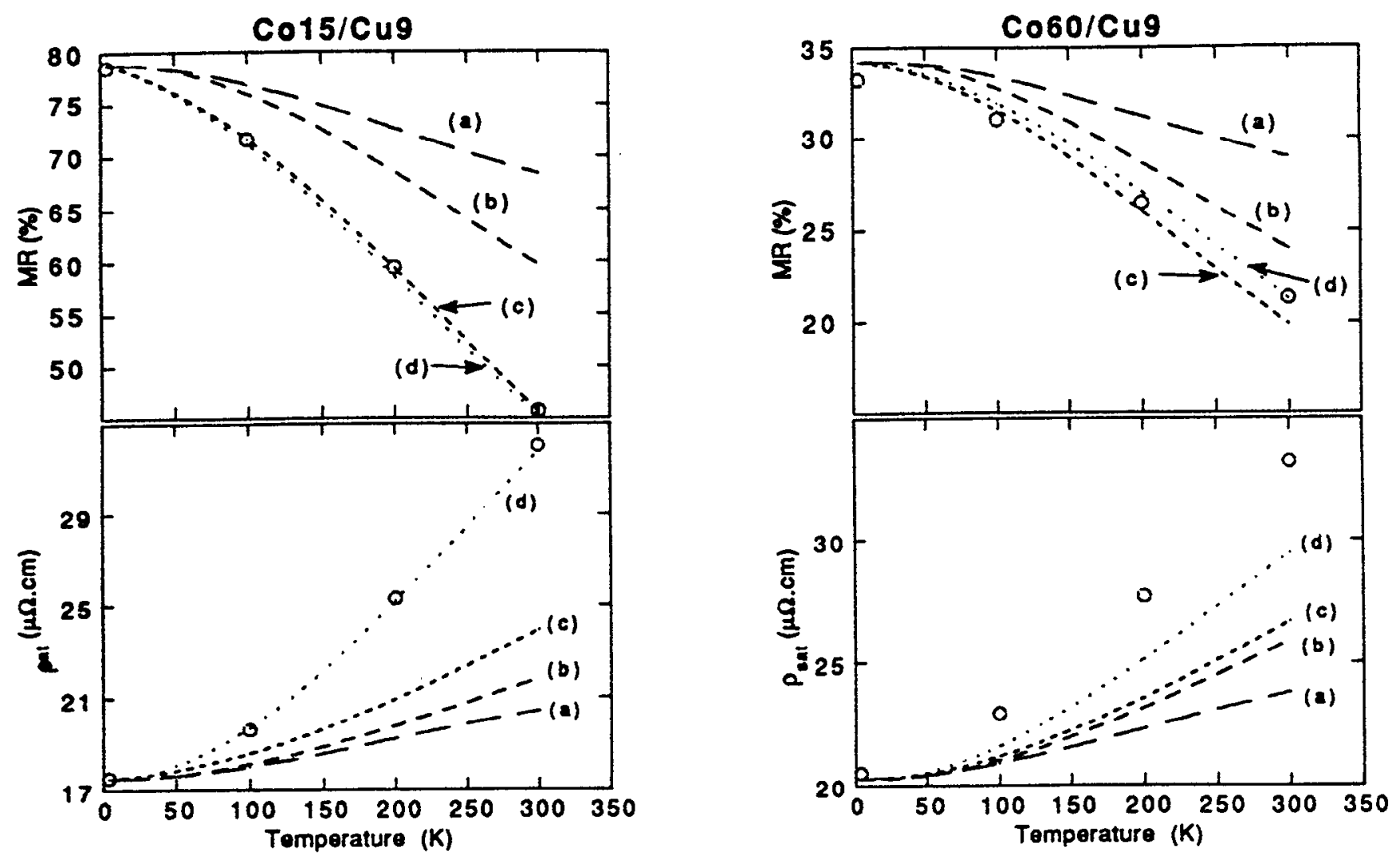

FIG. 2. Variation of the resistivity in the saturated state (bottom) and MR (top) as a function of temperature for (Co $15 \AA / C u 9 \AA)$ (left) and (Co $60 \AA / \mathrm{Cu} 9$ $\AA$ ). Circles: experimental data from Mosca et al. (see Ref. 13). Curve (a): calculation taking into account the phonon and magnon intrachannel resistivilies within $\mathrm{Co}$ and $\mathrm{Cu}$ layers. Curve (b): same calculation than (a) but also taking into account interchannel spin mixing terms within Co. Curves (c) and (d): calculation taking into account temperature-dependent scattering by the interfaces; (c) is calculated with an interchannel term; (d) is calculated with an intrachannel term.

the resistivity of Co $15 \AA / \mathrm{Cu} 9 \AA$; see curves (d) in Fig. 2 . The variation with $T$ we obtain for the resistivity of Co 60 $\AA / \mathrm{Cu} 9 \AA$ is slightly too small.

\section{DISCUSSION AND CONCLUSION}

Our results can be summarized as follows. We have found that introducing within layers the temperaturedependent (intrachannel) resistivity and spin-mixing terms derived from data in bulk materials is not sufficient to account for the variation of the resistivity and MR ratio with $T$ [the weak effectiveness of $\delta \rho_{\sigma}(T)$ to reduce the MR is partly due to its pronounced spin dependence in Co]. ${ }^{11}$ The best fits have been obtained by also taking into account temperaturedependent interfacial scattering. It is interesting to notice that the best fit is obtained by introducing intrachannel resistivity terms, and no interchannel spin mixing term in the interfacial zone. This can be ascribed to incoherent scattering by the spin fluctuations at the interfaces. The incoherence is consistent with spin fluctuation within quite disordered interfaces.

The final conclusion is that the temperature dependence of the resistivity and $\mathrm{MR}$ of $\mathrm{Co} / \mathrm{Cu}$ multilayers can be accounted for, at least approximatcly, by taking into account not only phonon and magnon resistivity terms determined in bulk materials, but also an additional contribution from interfaces.

\section{ACKNOWLEDGMENTS}

This work was supported in part by the European Economic Community [Esprit Project No. BRA6146) and by the CNRS Grant No. AI0693 (NSF-CNRS) collaboration].

${ }^{1}$ M. N. Baibich, J. M. Broto, A. Fert, F. Nguyen Van Dau, F. Petroff, P. Etienne, G. Creuzet, A. Friederich, and J. Chazelas, Phys. Rev. Lett. 61, 2472 (1988).

${ }^{2}$ R. E. Camley and J. Barnas, Phys. Rev. Lett. 63, 664 (1989).

${ }^{3}$ J. Barnas, A. Fuss, R. E. Camley, P. Grünberg, and W. Zinn, Phys. Rev. B 42, 8110 (1990).

${ }^{4}$ P. M. Levy, S. Zhang, and A. Fert, Phys. Rev. Lett. 65, 1643 (1990); S. Zhang, P. M. Levy, and A. Fert, Phys. Rev. B 45, 8689 (1992).

${ }^{5}$ A. Fert and I. A. Campbell, J. Phys. F 6, 849 (1976); I. A. Campbell and A. Fert, Ferromagnetic Materials, edited by E. P. Wohlfarth (NorthHolland, Amstcrdam, 1982), p. 760

${ }^{6}$ S. Zhang and P. M. Levy, Phys. Rev. B 43, 11048 (1991).

${ }^{7}$ M. Hasegawa, Phys. Rev. B 22, 15080 (1993).

${ }^{8}$ B. L. Johnson and R. E. Camley, Phys. Rev. B 44, 9997 (1991).

${ }^{9}$ The angular integration is stopped at an angle corresponding to $\cos \Theta_{c}=1 /$ $\sqrt{k_{F}}{ }^{*} \lambda$ before the direction parallel to the layer plane, which can be justified by arguing that if the electron lifetime is finite, the uncertainty principle prevents its velocity from being exactly parallel to the layers. For details, see A. Vedyayev, B. Dieny, and N. Ryshanova, Europhys. Lett. 19, 329 (1992).

${ }^{10}$ G. K. White and S. B. Woods, Philos. Trans. Ser. A 251, 273 (1959).

${ }^{11}$ B. Loegel and F. Gautier, J. Phys. Chem. Sol. 32, 2723 (1971).

${ }^{12}$ B. Dieny, Europhys. Lett. 17, 261 (1992).

${ }^{13}$ D. H. Mosca, F. Petroff, A. Fert. P. Schroeder, W. P. Pratt, Jr., R. Loloee, and S. Lequien, J. Magn. Magn. Mat. 94, L1 (1991).

${ }^{14}$ C. Mesny, P. Panissod, and R. Loloee, Phys. Rev. B 45, 12269 (1992). 